\title{
Inhibition of Paracoccidioides brasiliensis by ajoene is associated with blockade of phosphatidylcholine biosynthesis
}

Gioconda San-Blas, Julio A. Urbina, Edgar Marchán, Lellys M. Contreras, Françoise Sorais and Felipe San-Blas

Microbiology (1997), 143, 1583-1586

p. 1584 , Methods section

p. 1585, column headings in Tables 1 and 2

The concentrations of ajoene should read $25 \mu \mathrm{M}$ and $50 \mu \mathrm{M}$, not $25 \mathrm{mM}$ and $50 \mathrm{mM}$, respectively. 